\title{
Coherent control of selective bond breaking: HOD in the Ã-state revisited
}

\author{
Dey, Diptesh; Tiwari, Ashwani Kumar; Henriksen, Niels Engholm
}

Published in:

Chemical Physics Letters

Link to article, DOI:

10.1016/j.cplett.2018.12.017

Publication date:

2019

Document Version

Peer reviewed version

Link back to DTU Orbit

Citation (APA):

Dey, D., Tiwari, A. K., \& Henriksen, N. E. (2019). Coherent control of selective bond breaking: HOD in the Ãstate revisited. Chemical Physics Letters, 716, 131-133. https://doi.org/10.1016/j.cplett.2018.12.017

\section{General rights}

Copyright and moral rights for the publications made accessible in the public portal are retained by the authors and/or other copyright owners and it is a condition of accessing publications that users recognise and abide by the legal requirements associated with these rights.

- Users may download and print one copy of any publication from the public portal for the purpose of private study or research.

- You may not further distribute the material or use it for any profit-making activity or commercial gain

- You may freely distribute the URL identifying the publication in the public portal

If you believe that this document breaches copyright please contact us providing details, and we will remove access to the work immediately and investigate your claim. 


\title{
Coherent control of selective bond breaking: HOD in the $\tilde{A}$-state revisited
}

\author{
Diptesh Dey $^{\mathrm{a}}$, Ashwani K. Tiwari ${ }^{\mathrm{b}}$, Niels E. Henriksen ${ }^{\mathrm{a}, 1}$ \\ ${ }^{a}$ Department of Chemistry, Technical University of Denmark, Building 207, DK-2800 Lyngby, Denmark \\ ${ }^{b}$ Indian Institute of Science Education and Research Kolkata, Mohanpur 741246, India
}

\begin{abstract}
The VUV photodissociation dynamics of HOD in the first ( $\tilde{A}$-state) absorption band following a non-resonant impulsive vibrational excitation is studied theoretically. We show that a change in the linear temporal chirp of a VUV pulse with a fixed frequency distribution leads to control of the branching ratio $(\mathrm{H}+\mathrm{OD}) /(\mathrm{D}+\mathrm{OH})$ between the two fragmentation channels.

Keywords: Dynamic Stark effect, Pulse shaping, Coherent control
\end{abstract}

\section{Introduction}

Control of molecular processes based on the quantum mechanical principles of coherence and interference is a topic of current interest [1,2]. Coherent excitation can be accomplished by laser light and the phase modulation of the frequency components of a short laser pulse [3] leads to control of quantum interferences at the molecular scale.

Seminal theoretical work in this field (so-called bichromatic control) [4] envisioned two continuous wave lasers with appropriately chosen frequencies and a controllable relative phase; and it was shown that interference between different pathways could be controlled by the phase. To that end it should be noted that for gas-phase fragmentation, in the weak-field limit, coherent (interference) control of asymptotic fragment states is not possible if excitation starts from a single eigenstate. Thus, a key pre-requisite for coherent control is that the system can be driven to a given energy level via different

URL: neh@kemi . dtu.dk (Niels E. Henriksen) 
pathways; in the weak-field limit this can be accomplished for pulsed laser excitation with a bandwidth that encompasses more than one eigenstate state in an initial superposition state $[2,5]$.

As a prototype of the chemically interesting scenario of bond-selective chemistry, the control of the branching ratio (in the $\tilde{A}$-state) between the two fragmentation channels in $\mathrm{HOD}(\mathrm{H}+\mathrm{OD}$ or $\mathrm{D}+\mathrm{OH})$ has been a matter of long-term interest $[6,7,8,9$, $10,11,12,13,14,15]$. To that end, we mention previous work assuming, oriented HOD molecules [10], the creation of a vibrational superposition state by an unspecified mechanism [11], or a scheme which required non-trivial pulse shaping of intense ultrashort vacuum-ultraviolet (VUV) pulses [14]. The aim of this letter is to investigate a control scheme which allows for a more feasible experimental implementation and which demonstrate coherent (interference) control in its purest form.

The present work is inspired by recent experimental work on HOD which employed, respectively, (transform limited) $16 \mathrm{fs}$ VUV pulses at $161 \mathrm{~nm}$ [16] and fewcycle pulses at $800 \mathrm{~nm}$ [17]. We showed recently that such few-cycle pulses are well suited for non-resonant vibrational excitation of HOD [15].

Branching ratio control can, e.g., be accomplished via control of the time-delay between the non-resonant pulse and an ultrashort VUV pulse [15]. The shift/delay of a laser pulse in time corresponds to a linear phase modulation in the spectral regime. In this letter, we consider an alternative type of phase modulation, leading to a linear temporal chirp of the VUV pulse [5] at a fixed time delay.

\section{Theory and computation}

The dynamics of the HOD molecule is described within the two-degree-of-freedom model for the interatomic motion (see, e.g., Ref. [10]) employed in several previous publications, where the bending angle is fixed at $104.52^{0}$. The non-resonant vibrational excitation is considered for randomly oriented HOD molecules, e.g., corresponding to the rotational ground state of HOD [15]. A constant electronic transition dipole moment is employed.

Within first-order perturbation theory for the light-molecule interaction, the nuclear 
wave packet in the electronically excited state can be expressed by

$$
\left.\chi_{2}(t)\right\rangle=\frac{i}{\hbar} \int_{t_{0}}^{t} d t^{\prime} e^{-i \hat{H}_{2}\left(t-t^{\prime}\right) / \hbar} E\left(t^{\prime}\right) e^{-i \hat{H}_{1}\left(t^{\prime}-t_{0}\right) / \hbar}\left|\chi_{1}\left(t_{0}\right)\right\rangle
$$

where $\hat{H}_{1}$ and $\hat{H}_{2}$ are the nuclear Hamiltonians of the two electronic states, $E(t)$ is the electric field of the laser pulse, and $\left\langle\chi_{1}\left(t_{0}\right)\right\rangle$ is the nuclear wave packet of HOD at time $t_{0}$, times the projection of the electronic transition dipole moment in the direction of the polarization vector of the plane-polarized field. Numerical wave packet propagation is carried out on a two-dimensional grid (see, e.g., Ref. [15]).

$\left\langle\chi_{1}\left(t_{0}\right)\right\rangle$ is created by non-resonant vibrational excitation of $\operatorname{HOD}[15,18,19,20]$ where the mechanism can be described as dynamic Stark effect or, equivalently, as impulsive Raman scattering. The non-resonant vibrational excitation from the vibrational ground state of HOD, employs a train of six $800 \mathrm{~nm}$ pulses. The time duration of each pulse is $3.5 \mathrm{fs}$ and the spacing between the pulses is $12.2 \mathrm{fs}$, corresponding to the vibrational period of the O-D bond. As shown in Ref. [15], this leads to an essentially selective vibrational excitation of the O-D bond. With this pulse train, the excited state population is approximately $10 \%$, i.e., the vibrational excitation is relatively modest.

The electric field $E(t)$ of the VUV transition is a Gaussian pulse centered at the frequency $\omega_{0}$ and with a variable linear temporal chirp, that is [5],

$$
E(t)=E_{0} \operatorname{Re}\left[\sqrt{\frac{\tau_{0}^{2}}{\tau_{0}^{2}-i \beta_{0}}} \exp \left(-\frac{t^{2}}{2 \tau^{2}}-i \beta t^{2} / 2-i \omega_{0} t\right)\right]
$$

with pulse duration $\tau$ and linear temporal chirp $\beta$ ( $t$ is replaced with $t-t_{c}$ when the pulse is centered at $t=t_{c}$ ). These parameters are related to the frequency bandwidth $1 / \tau_{0}$ and the quadratic spectral chirp $\beta_{0}$ via $\tau^{2}=\tau_{0}^{2}\left(1+\beta_{0}^{2} / \tau_{0}^{4}\right)$ and $\beta=\beta_{0} /\left(\tau_{0}^{4}+\beta_{0}^{2}\right)$, respectively. For $\beta_{0}=0, \beta=0$ and $\tau=\tau_{0}$ and the result is a transform-limited Gaussian pulse. In the following, we fix the frequency bandwidth (corresponding to a given temporal duration of the transform-limited pulse) and modulate the quadratic spectral chirp $\beta_{0}$ leading to different values of $\tau$ and $\beta$. The pulse is centered at $t=t_{c}$ relative to the peak in the last (sixth) pulse of the non-resonant pulse train, such that the pulse train and the VUV pulse do not overlap after the introduction of the chirp. 


\section{Results and discussion}

The center wavelength of the VUV field is chosen to $166.4 \mathrm{~nm}$, corresponding to the maximum of the absorption band, and the amplitude $E_{0}$ is chosen such that the excitation probability is less than a few percent.

Figure 1 shows the branching ratio as a function of the quadratic spectral chirp $\beta_{0}$ and the full width at half maximum (FWHM) of the unchirped pulse $\left(\beta_{0}=0\right)$. The VUV pulse is centered at $t_{c}=302.8 \mathrm{fs}$ relative to the peak in the last (sixth) pulse of the non-resonant pulse train. We observe a modulation of the branching ratio as a function of chirp and the modulation depends clearly also on the bandwidth (FWHM) of the unchirped pulse. Thus, for pulse durations larger than about $15 \mathrm{fs}$, the effect of chirp almost vanishes. The bandwidth is then too small to allow for interference between the different vibrational states in the initial superposition state. The modulation is largest for the shortest pulses. For example, for FWHM $=5 \mathrm{fs}$, we observe that the branching ratio increases from 1.9 to 2.35 as a function of the chirp. The results are shown for chirps $\left|\beta_{0}\right| \leq 50 \mathrm{fs}^{2}$, and are essentially unchanged for larger values of $\left|\beta_{0}\right|$. When one full vibrational period of the O-D oscillation is added to the time delay $t_{c}$, we obtain essentially the same result (due to essentially stationary O-H oscillations).

Figure 2 shows the branching ratio, now with the VUV pulses shifted by $t_{c}+$ $(1 / 2) P_{\mathrm{OD}}$ where $P_{\mathrm{OD}}$ is the vibrational period of O-D. The relative phases in the initial vibrational superposition state will now differ from the previous case. For example, for $\mathrm{FWHM}=5 \mathrm{fs}$, we observe that the branching ratio decreases from 2.8 to 2.35 as a function of the chirp, i.e., the opposite trend compared to Fig. 1. The modulation as a function of bandwidth (FWHM) is similar to the results in Fig. 1.

Figure 3 shows the branching ratio, now with the VUV pulses shifted to $t_{c}+$ (3/4) $P_{\mathrm{OD}}$ where $P_{\mathrm{OD}}$ is the vibrational period of O-D. For FWHM $=5$ fs, we observe that the branching ratio decreases from 3.26 to 2.33 as a function of the chirp. Especially for the shortest pulses, we observe a noticeable asymmetry with respect to positive or negative values of $\beta_{0}$.

Overall, we notice a potentially quite large modulation of the branching ratio, even with a quite modest population in excited vibrational states, i.e, of the order of $10 \%$. 


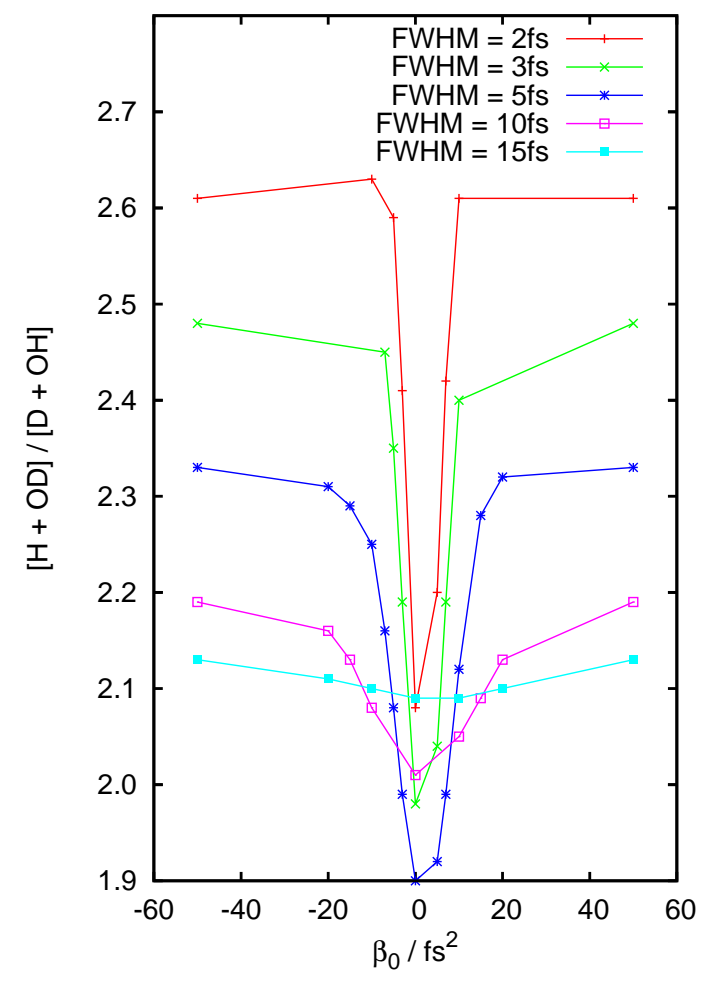

Figure 1: Branching ratio as a function of quadratic spectral chirp $\beta_{0}$ and the FWHM of the unchirped $\left(\beta_{0}=0\right)$ VUV pulse. The time delay between the non-resonant pulse train and the VUV-pulse is $t=t_{c}$ (see text for details). The calculated points for a given FWHM are connected by straight lines in order to guide the eye. 


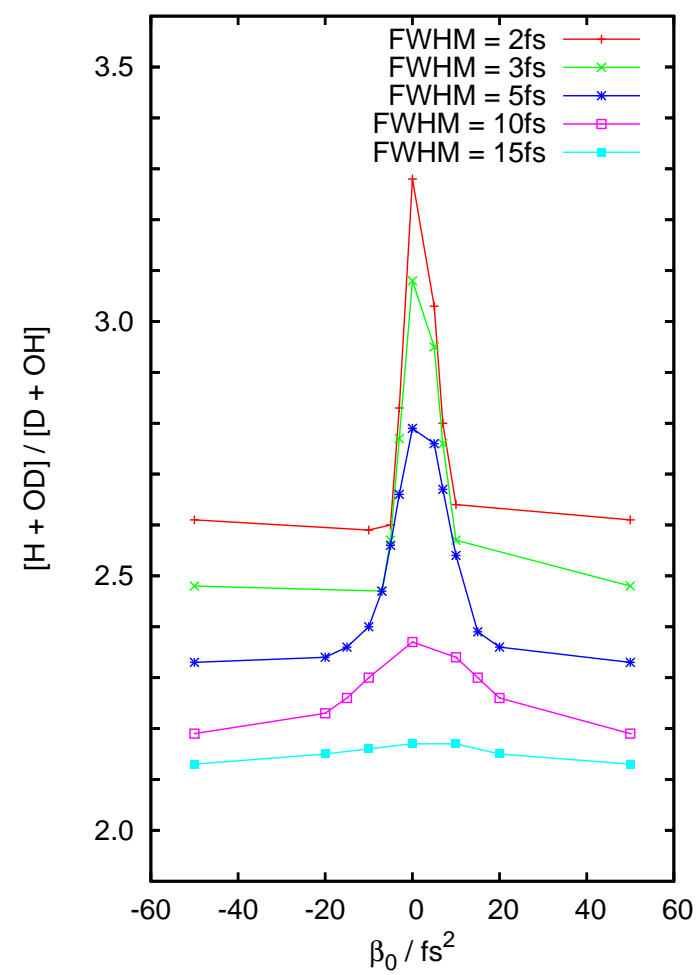

Figure 2: Branching ratio as a function of quadratic spectral chirp $\beta_{0}$ and the FWHM of the unchirped $\left(\beta_{0}=0\right)$ VUV pulse. One-half of a vibrational O-D period has been added to the time delay $t_{c}$ in Fig. 1 (see text for details). 


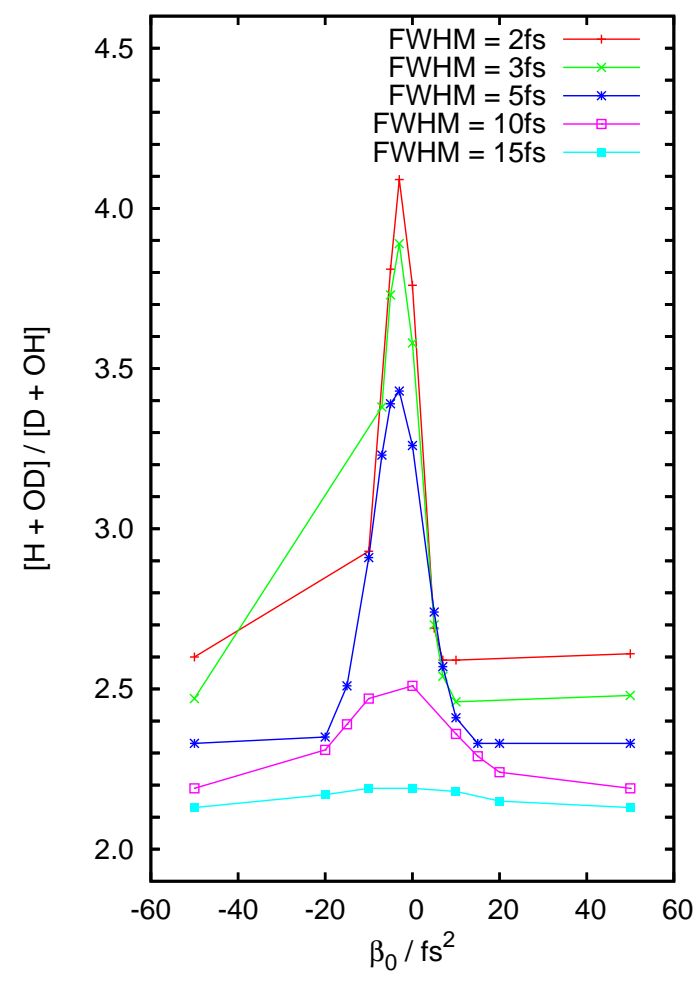

Figure 3: Branching ratio as a function of quadratic spectral chirp $\beta_{0}$ and the FWHM of the unchirped $\left(\beta_{0}=0\right)$ VUV pulse. Three-quarter of a vibrational O-D period has been added to the time delay $t_{c}$ in Fig. 1 (see text for details). 
We observe a modulation of the branching ratio with respect to chirp or time delay that: (i) decreases as a function of increased pulse duration (reduced bandwidth $1 / \tau_{0}$ ); (ii) for a fixed pulse duration (bandwidth $1 / \tau_{0}$ ) is of similar magnitude with respect to chirp or time delay. Thus, there is clearly close similarities between "frequency-domain" (chirp) and "time-domain" (time delay) control. The latter can also be considered as a linear spectral chirp in the frequency domain.

The dependence of the branching ratio on the time delay is more readily explained [15] than the dependence on the quadratic spectral chirp or, equivalently, the linear temporal chirp which corresponds to a time-dependent VUV excitation frequency. Thus, the dependence on the time delay for $\beta_{0}=0$ is related to the different position/momentum distributions of the O-D bond at the time of the VUV excitation [15] whereas the dependence of the chirp is a quantum interference effect without a simple classical picture.

Although the branching ratios reported in this work are all larger than 1.9, a more detailed scanning of time delays and chirps give branching ratios which are still not smaller than about 1.7. Smaller branching ratios, e.g., corresponding to preferred breaking of the O-D bond can be obtained when the initial (non-stationary) vibrational state contains a higher population of excited states. This will allow for higher vibrational amplitudes/momenta ("initial localization of the O-D bond") in time-delay control or more comprehensive quantum interference control in chirped-pulse excitation at a fixed time delay.

In conclusion, with chirped pulses originating from transform-limited VUV pulses, slightly shorter than the ones employed in Ref. [16], it should be possible to demonstrate genuine coherent control of the selective bond breaking in HOD.

\section{Acknowledgments}

The research leading to these results has received funding from the European Union's Horizon 2020 research and innovation programme under the Marie Sklodowska-Curie Grant agreement No. 713683 (COFUNDfellowsDTU).

\section{References}


[1] C. Brif, R. Chakrabarti, H. Rabitz, New J. Phys. 12 (2010) 075008.

[2] M. Shapiro, P. Brumer, Principles of the Quantum Control of Molecular Processes (Wiley, New York, 2012).

[3] A.M. Weiner, Optics Comm. 284 (2011) 3669.

[4] P. Brumer, M. Shapiro, Chem. Phys. Lett. 126 (1986) 541.

[5] A.K. Tiwari, N.E. Henriksen, J. Chem. Phys. 144 (2016) 014306.

[6] V. Engel, R. Schinke, J. Chem. Phys. 88 (1988) 6831.

[7] D.G. Imre, J. Zhang, Chem. Phys. 139 (1989) 89.

[8] R.L. Vander Wal, J.L. Scott, F.F. Crim, J. Chem. Phys. 92 (1990) 803.

[9] I. Bar, Y. Cohen, D. David, S. Rosenwaks, J.J. Valentini, J. Chem. Phys. 93 (1990) 2146.

[10] B. Amstrup, N.E. Henriksen, J. Chem. Phys. 97 (1992) 8285.

[11] N.E. Henriksen, B. Amstrup, Chem. Phys. Lett. 213 (1993) 65.

[12] M. Shapiro, P. Brumer, J. Chem. Phys. 98 (1993) 201.

[13] N. Elghobashi, P. Krause, J. Manz, M. Oppel, Phys. Chem. Chem. Phys. 5 (2003) 4806.

[14] A.K. Tiwari, K.B. Møller, N.E. Henriksen, Phys. Rev. A 78 (2008) 065402.

[15] D. Dey, N.E. Henriksen, J. Chem. Phys. 148 (2018) 234307.

[16] A. Baumann, S. Bazzi, D. Rompotis, O. Schepp, A. Azima, M. Weiland, D. Popova-Gorelova, O. Vendrell, R. Santra, M. Drescher, Phys. Rev. A 96 (2017) 013428 .

[17] D. Mathur, K. Dota, D. Dey, A.K. Tiwari, J.A. Dharmadhikari, A.K. Dharmadhikari, J. Chem. Phys. 143 (2015) 244310. 
[18] B.J. Sussman, D. Townsend, M.Y. Ivanov, A. Stolow, Science 314 (2006) 278.

[19] D. Townsend, B.J. Sussman, A. Stolow, J. Phys. Chem.A 115 (2011) 357.

[20] E.F. Thomas, N.E. Henriksen, J. Chem. Phys. 144 (2016) 244307. 